\title{
Multimode Interference Filter to solve degradation on Couplers Common-Mode Rejection
}

\author{
C. Vázquez ${ }^{1}$, C. Aramburu ${ }^{2}$, M. López-Amo', J.M.S. Pena ${ }^{1}$ \\ ${ }^{1}$ Dpto. Ingeniería Eléctrica, Electrónica y Automática, Area Tecnología Electrónica, Universidad Carlos III, \\ Butarque 15, Leganés 28911, Madrid, Spain • Fax: 3416249429•Phone: 3416249430•E-mail: cvazquez@ing.uc3m.es \\ ${ }^{2}$ Dpto. Ingeniería Eléctrica y Electrónica, Universidad Pública de Navarra, \\ Campus de Arrosadia, s/n, 31006 Pamplona (Navarra)•E-mail: candido@si.upna.es
}

\begin{abstract}
Introduction
Balance detection is critical in Frequency Division Multiple Access Networks (FDMA) that utilize coherent technology and in certain sensor applications. Compact monolithic integration can be achieved combining detectors with $3 \mathrm{~dB}$ multimode interference (MMI) couplers [1] having high common-mode rejection ratios (CMRR) [2-3]; main feature because this parameter quantifies local oscillation noise reduction [4]. Additional advantages of these couplers include: fabrication tolerances, insensibility to polarization and low insertion loss. As a result these couplers have also been used in other Photonic Integrated Circuits (PIC) [2,5,9]. But in none of these applications influence of partially exciting first order TE01 mode in the access waveguides has been analyzed. This last effect can be present when strong confined waveguides, imposed by active devices design such as lasers in the PIC, are used and cost effective photolithography fabrication process fix a limitation in width of access waveguides[8]. In this paper, after quantifying degradation of CMRR 3dB-coupler due to excitation of TE01 mode, a novel compact circuit including MMI Coupler+Bend+MMI-Filter (CBF) is proposed. We show CBF circuit has better CMRR at the expense of moderate loss. A completely tolerance analysis to main geometrical parameters has been also carried out.
\end{abstract}

Filter and Coupler+Bend Design

In this section we are going to describe independent design and tolerance analysis for each element of the $C B F$ circuit. Its layout is shown in Fig. 1. $0,27 \mu \mathrm{m}$ thick InP/InGaAsP buried waveguides are used. This strong guiding structure allows monolithic integration and compact size. Input and output waveguides are $2 \mu \mathrm{m}$ width so two modes are confined.

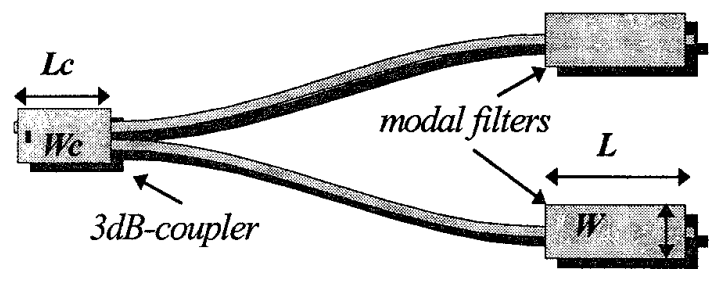

Figure 1: Coupler and Filter upper view with Bends

It has been shown in $[6,3]$ that restricted interference $3 \mathrm{~dB}$ couplers exhibit higher fabrication tolerances in shorter devices. So our 3-dB coupler is $12 \mu \mathrm{m}$ wide (Wc) and $230 \mu \mathrm{m}$ long (Lc) with inputs located at $\mathrm{Wc} / 3$ and $2 \mathrm{Wc} / 3$. Beam Propagation Method (BPM) is used in the design to include bends waveguides. To reduce our problem from three to two dimensions, effective index method was perform, $n_{f}$ is MMI section effective index. Curve guides are $1070 \mu \mathrm{m}$ long to assure no loss penalty although shorter bends could have been used. Our Coupler+Bend device exhibits a CMRR of $-33,5 \mathrm{~dB}$ and $0,2 \mathrm{~dB}$ insertion loss, $\gamma$. Including bends in simulation is fundamental because CMRR changes. Designing with two confined modes in input/output guides suggest us the need to analyzed effect of TE01 mode in coupler performance. For doing so, we have considered two ways of input waveguide excitation denoted: (a) excitation mono (100\% TE00 mode injection), (b) excitation bi ( $90 \%$ TE00 $+10 \%$ TE01 mode injection). We observe that in case (b) CMRR degrades to $-17,6 \mathrm{~dB}$ and $\gamma$ increases to $0,96 \mathrm{~dB}$. To solve this problem a multimode filter is designed.

Guided modes, $m$, in MMI section depend on input profile [2]. Table I. shows normalized phases difference between fundamental and $m$ mode, $\phi^{\prime}=\left|\phi_{\mathrm{m}} /\left(\mathrm{L} / \mathrm{L}_{1}\right)\right|$ at length $\mathrm{L}$, for different injection cases, with $\mathrm{L}_{1}$ defined in [7] as $4 \mathrm{n}_{\mathrm{f}} \mathrm{W}^{2} / \lambda$. Row 2 shows $\phi^{\prime} \mathrm{m}$ in case of fundamental mode injection so only pair modes are excited. While if first mode is injected, impair modes are excited as is shown in row 3. In multimode devices self-imaging occurs at lengths where different eigenmodes interfere

\begin{tabular}{ccccc}
\hline Input & $\begin{array}{c}\text { MMI mode } \\
\text { number, } \mathbf{m}\end{array}$ & $\phi_{\mathbf{m}}^{\prime}=\left(\mathrm{m}^{2}+2 \mathrm{~m}\right) \pi$ & $\mathbf{L}$ & $\begin{array}{c}\text { Image } \\
\text { type }\end{array}$ \\
\hline $\mathrm{TE}_{00}$ mode & $0 / 2 / 4 \ldots$ & $0 / 8 \pi / 24 \pi .$. & $\mathrm{q} \mathrm{L}_{1} / 4$ & $\begin{array}{c}\text { Self- } \\
\text { image }\end{array}$ \\
$\mathrm{TE}_{01}$ mode & $1 / 3 / \ldots$ & $3 \pi / 15 \pi \ldots$ & $\begin{array}{c}\mathrm{L}_{1} / 12+ \\
\mathrm{L}_{1}(\mathrm{r}-1) / 6\end{array}$ & $\begin{array}{c}\text { Two-fold } \\
\text { images }\end{array}$ \\
\hline
\end{tabular}
constructively, while two-fold images are obtained at $1 / 4$ of that length [7]. Applying those principals, device lengths show in row 4 are obtained, where $q$ and $r$ are integer numbers and modes 5,11 are not excited. MMI modal filter transmits TE00 mode and rejects TE01 mode, so filtering is achieved when:

$$
q L_{1} / 4=L_{1} / 6\left(1 / 2+L_{1}(r-1)\right)
$$

Table 1. Filter phases difference and lengths depending on injection. 
First length satisfying (1) is $L=L_{1} / 4$, with $\mathrm{q}=1, \mathrm{r}=2$. The same result is obtained using expressions derived in [9]. Filter tolerances analysis reveals that wider devices have better rejection ratios, $R$, to TE0I mode as shown in Fig. 2, where Lmmi is filter length and $\mathrm{R}=10 \log \left(\mathrm{P}_{\mathrm{TE} 00} / \mathrm{P}_{\mathrm{TE}} 01\right)$.

\section{CBF Circuit Design and Tolerances Analysis.}

Results described in previous section reveals the need to concatenated coupler+bend and filter to overcome CMRR $3-\mathrm{dB}$ coupler degradation when injecting TE01 mode. Layout of this new circuit is shown in Fig. 1. 12 $\mu \mathrm{m}$ filter width, $346 \mu \mathrm{m}$ long is selected to assure higher rejection ratio within the whole tolerance range (see Fig. 2). BPM Field propagation, excitation mono, can be seen in Fig. 3.

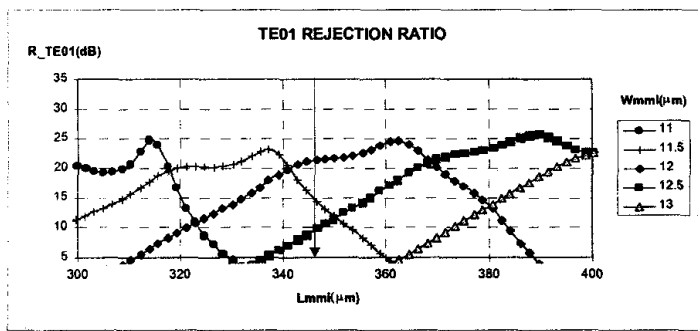

Figure 2: Multimode Filter fabrication tolerances.

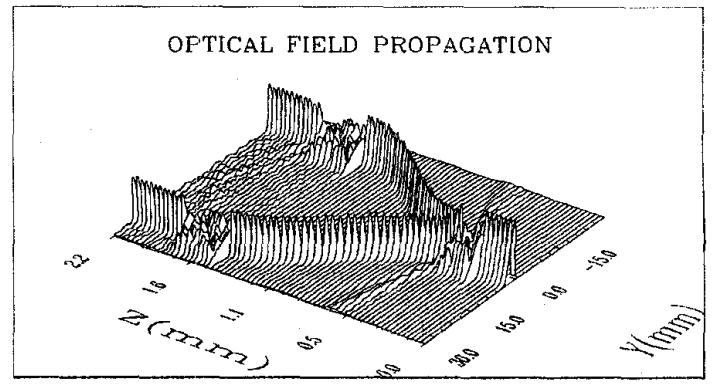

Figure 3: Field distribution along CBF circuit.

Fabrication tolerances at both excitations, mono and bi are simulated. Design target was CMRR better than -20dB. Fig. 4 results reveal CMRR improvement of CBF circuit compare with no filter presence in fabrication tolerances. At 0 error, excitation

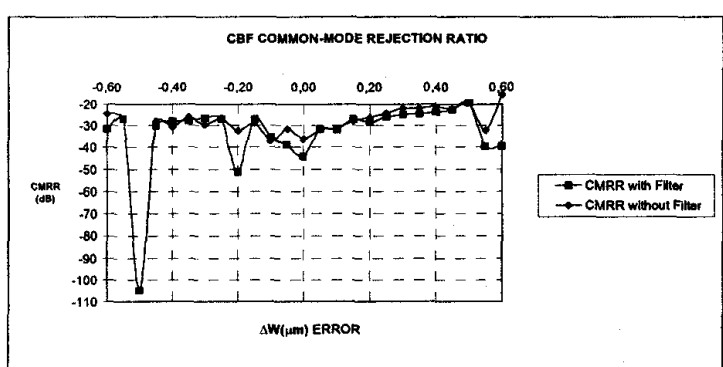
mono, has $10 \mathrm{~dB}$ improvement with $0,2 \mathrm{~dB}$ loss. If excitation $\boldsymbol{b i}$ is considered CMRR in CBF circuit improves from $-17,4 \mathrm{~dB}$ to $30 \mathrm{~dB}$; with $0,5 \mathrm{~dB}$ loss increase. Loss penalty can be overcome via amplification or using tapers at access bends.

Figure 4: CMRR width tolerance in CBF circuit (excitation mono)

\section{Conclusions}

Novel CBF circuit overcomes first mode effect on CMRR degradation of couplers used in balance coherent detection receivers. CMRR improves in $12 \mathrm{~dB}$ when partially injecting first mode access guide and in $10 \mathrm{~dB}$ if injecting fundamental mode. Circuit has $\pm 0,4 \mu \mathrm{m}$ width tolerance, most critical parameter. Filter width can be reduced to shorter circuit if less CMRR tolerance range is needed. General interference can be used in other $\mathrm{CBF}$ circuits.

\section{References}

/1/ R. J. Deri, et al. "Ultracompact monolithic integration of balanced, polarization diversity photodetectors for coherent lightwave receivers", IEEE Photonic Technology Letters, pp. 1238-1240, (1992).

$12 /$ L. B. Soldano and E. C. M. Pennings, "Optical Multi-Mode Interference Devices Based on Self-Imaging: Principles and Applications", J. Lightwave Tech., pp.615-627, (1995).

13/ C. Vázquez, F. J. Mustieles, F. Hernández-Gil, "Three-Dimensional Method for Simulation of Multimode Interference Couplers", J. Lightwave Tech., pp.2296-2299, (1995).

14/ R. J. Deri, R. Welter. "Effect of Capacitance imbalance on Common-Mode rejection of balanced photodetector pairs", El. Lett., pp.75-76, (1993).

15/ M. R. Paiam, R. Y. MacDonald. "A 12-Channel Phased-Array Wavelength Multiplexer with Multimode Interference Couplers", IEEE Photonic Technology Letters, pp. 241-243, (1998).

16/ P. A. Besse, M. Bachmann, H. Melchior, L. B. Soldano and M. K. Smit, "Optical Bandwith and Fabrication Tolerances of Multimode Interference Couplers". J. Lightwave Technology, 12, pp. 1004-1009, (1994).

$17 /$ R. Ulrich and T. Kamiya, "Resolution of self-images in planar optical waveguides", J. Opt. Soc. Am., pp. 583-592, (1978).

18/ C.Vázquez, C.Aramburu, M. López-Amo, J. M. S. Pena. "Accessing guides first order mode influence and optimize tolerances in multimode interference couplers". ICAPT'98, Otawa (to be published).

19/ M. Bachman, P. A. Besse, H. Melchior. "Overlapping-image multimode interference couplers with a reduced number of self-images for uniform and nonuniform power splitting". Appl. Optics., pp.6898-6910, (1995). 